\title{
The effect of leisure activity golf practice on motor imagery: an fMRI study in middle adulthood
}

\author{
Ladina Bezzola $^{1,2 *}$, Susan Mérillat ${ }^{2}$ and Lutz Jäncke ${ }^{1,2}$ \\ Division of Neuropsychology, Institute of Psychology, University of Zurich, Zurich, Switzerland \\ 2 International Normal Aging and Plasticity Imaging Center (INAPIC), University of Zurich, Zurich, Switzerland
}

\author{
Edited by: \\ Shuhei Yamaguchi, Shimane \\ University, Japan \\ Reviewed by: \\ Rachael D. Seidler, University of \\ Michigan, USA \\ Thomas F. Münte, University of \\ Magdeburg, Germany \\ ${ }^{*}$ Correspondence: \\ Ladina Bezzola, Institute of \\ Psychology/Neuropsychology, \\ University of Zurich, \\ Binzmuehlestrasse 14/25, CH-8050 \\ Zurich, Switzerland. \\ e-mail: I.bezzola@psychologie.uzh.ch
}

\begin{abstract}
Much is known about practice-induced plasticity of the motor system. But it is not clear how a physical training influences the mental rehearsal of the practiced task and its associated hemodynamic responses. In the present longitudinal study with two measurement time-points, we used the method of functional magnetic resonance imaging ( $f M R I)$ and a motor imagery task, in order to explore the dynamic neuro-functional changes induced by a highly complex physical training. The 11 golf novices between the age of 40 and 60 years practiced the motor training as leisure activity. Additionally, data from an age and sex-matched control group without golf training was collected. As a main result, we demonstrate that changes between the two measurement time-points were only found in the golf novice group. The golf novices showed a decrease in hemodynamic responses during the mental rehearsal of the golf swing in non-primary motor areas after the $40 \mathrm{~h}$ of golf practice. Thus, the results indicate that a complex physical leisure activity induces functional neuroplasticity in the seldom studied population of middle-aged adults, and that this effect is evident during mental rehearsal of the practiced task. This finding supports the idea that (a) a skill improvement is associated with a modified activation pattern in the associated neuronal network that can be identified during mental rehearsal of the practiced task, and that (b) a strict training protocol is not necessary to induce functional neuroplasticity.
\end{abstract}

Keywords: motor imagery, fMRI, middle adulthood, functional neuroplasticity, motor learning

\section{INTRODUCTION}

The brain shows an enormous capacity to adapt rapidly and efficiently to new requirements and experiences during the process of learning. This capacity is reflected in structural adaptations (Draganski and May, 2008). Closely related with these neuroanatomical changes are the similarly important changes in neural recruitment patterns (Pascual-Leone et al., 2005; Jancke, 2009). Structural and functional neuroplasticity may be regarded as two processes with a mutual interaction. Meaning, that morphologic changes are accompanied by functional changes and vice-versa (Ilg et al., 2008; Granert et al., 2011). With respect to the different methodological approaches to study neuroplasticity, however, there is a practical differentiation based on the spatial and temporal methodological characteristics. Recent studies from the field of motor skill learning indicate that training-induced structural and functional adaptations occur across the whole lifespan (Seidler, 2007; Lustig et al., 2009). Given the clear focus of these studies on young adults or adults aged over 65 , the group of middle-aged adults has been neglected, meaning that our knowledge about age-related differences in both the extent and the pattern of training-induced adaptations across the lifespan is still limited.

There is conclusive evidence that the brain relies on the involvement of a widely distributed network including cortical and sub-cortical motor areas, cerebellum, and prefrontal areas, independently of age and particularly during the early stages of skill learning (Doyon and Benali, 2005; Halsband and Lange, 2006). As skill improves, the involvement of these neuronal regions changes. Longitudinal studies with a short training duration (i.e., several days) indicate a slowly developing activation increase at early stages of training (Karni et al., 1995; Ungerleider et al., 2002; Hlustik et al., 2004). In contrast, motor experts usually show smaller hemodynamic responses within the sensorimotor system compared with novices performing the same motor task (Jancke et al., 2000; Koeneke et al., 2004). This effect of expertise is considered to reflect increased efficiency and, hence, reduced neural costs in the neural networks controlling the practiced movements and skills. In addition, less cognitive control (i.e., in terms of reduced attentional demands) is required when a movement is well learned or even automatized (Godde and Voelcker-Rehage, 2010). There is thus far no conclusive study indicating when the transition from increased to decreased neural effort actually occurs.

Most studies on motor learning have registered the neural response to various kinds of finger movements because they are (1) easily performed within the confines of the MR scanner and (2) are not associated with large movement artifacts correlated with the experimental paradigm. To study more complex movements that include several joints and muscles or different body parts such as required for a golf swing, an alternative approach 
has used the method of motor imagery (Milton et al., 2008). The neuronal networks associated with the imagery of a particular movement from a first-person perspective are strikingly similar to those associated with movements that are overtly executed (Gerardin et al., 2000; Jeannerod, 2001; Lacourse et al., 2005). Several studies have provided strong evidence that traininginduced adaptations occur in both types of training protocols, that is, in mentally imagined practice (Olsson et al., 2008a,b; Page et al., 2009) and in physically executed practice (Doyon and Benali, 2005).

In addition to the type of training (overtly performed vs. mental imagery-based), several other training characteristics presumably influence the extent of training-related improvement in performance and associated neural changes. In the motor domain, the preceding longitudinal training studies in healthy subjects strictly applied experimental control in the training situations-keeping training parameters as constant as possible (e.g., training duration per day, overall training duration, training schedule, strategies, etc.). Only few studies have thus far compared different training parameters in terms of their impact on neural plasticity (Studer et al., 2010), while there are no longitudinal studies on the neural effects of a training performed in an environment outside experimental laboratory situations such as during leisure activity. Given that the participation in leisure activities influences the process of brain aging and the risk of developing dementia (Verghese et al., 2003; Rovio et al., 2005), it is important to extend previous research by focusing on training that approximates the real-life situation and has, therefore, higher ecological validity (Bezzola et al., 2011).

The present longitudinal functional magnetic resonance imaging (fMRI) study was, therefore, designed to investigate changes of neuronal recruitment while subjects mentally rehearsed a golf swing that had been overtly practiced between the two fMRI-based measurement time-points. Given the complexity of a golf swing, which requires considerable cognitive and motor control, an extended neural network including cortical, subcortical motor areas, and parietal regions was expected to be associated with mental rehearsal of the golf swing. Our primary interest concerned changes of neuro-functional recruitment patterns in association with golf training, such as activation increases/decreases in the involved brain regions, the recruitment of additional neural resources or the non-engagement of brain regions that were recruited before practice. Several studies (Solodkin et al., 2004; Milton et al., 2007; Guillot et al., 2008; Ionta et al., 2010) show that the neural activations during mental rehearsal of motor tasks are very similar to the activations identified during physical practice of the same task. Accordingly, expertise effects have been shown to occur during motor imagery (Fourkas et al., 2008) and motor execution (Jancke et al., 2000) of the expertise related motor task. These expertise effects were reflected in a more efficient neuronal network, i.e., an increased facilitation of the motor system and a reduced hemodynamic response. Thus, we hypothesized that the neural activations during mental rehearsal of the golf swing will change, in terms of a reduced hemodynamic response, as a consequence of golf practice.

\section{METHODS \\ PARTICIPANTS}

Twenty-two healthy volunteers (18 women, 4 men) with a mean age of $51.2(\mathrm{SD}=7.2$ ) years participated in the present study. A previous study investigating the structural correlates of traininginduced plasticity included the same study population (Bezzola et al., 2011). None of the participants had any history of neurological or psychiatric disorder. The study sample consisted of the following two groups, a golf novice group ( $n=11 ; 9$ women, 2 men) and an age-, sex, and handedness-matched control group $(n=11)$. Handedness was verified by means of the Annett Handedness questionnaire (Annett, 1970). According to this test, 20 participants (10 golf group, 10 control group) were classified as consistent right-handers and two participants as consistent lefthanders. All subjects gave written informed consent and the local ethics committee approved the present study, in compliance with the Helsinki Declaration.

\section{EXPERIMENTAL DESIGN}

A longitudinal study design with two measurement time-points was used. The golf pre-experience of golf group amounted to $77 \mathrm{~h}$. The interval between the two measurements referred to the golf group and amounted to 40 golf training hours. Each golf novice had an individual inter-measurement interval, calculated on the basis of the training hours, recorded on a training log. Since each participant of the golf group was matched (age, sex, handedness) to a participant of the control group, we ensured that the two groups had the same inter-measurement interval.

\section{TASK}

At both scan time-points, participants performed a kinetic motor imagery task. Before each scan session, participants were instructed about the task and advised to perform the imagery task from a first-person perspective, i.e., kinetic motor imagery. We used a block design with three conditions: (1) video condition with a slow motion golf swing ( $9.7 \mathrm{~s}$ ) of a professional golf player; (2) motor imagery condition with the mental rehearsal of the own golf swing, i.e., kinetic motor imagery; (3) control condition with a fixation cross lasting as long as the video condition (9.7 s). Each participant watched four epochs of the video sequence. Five imagery conditions followed every video sequence, meaning that each participant performed 20 imagery trials (conditions). The participants were instructed to imagine with their eyes closed their own golf swing from a first-person perspective and in slow motion, i.e., approximately $10 \mathrm{~s}$. Each participant marked for each trial the beginning and the end of the imagery condition by a button press. The visual presentation and the collection of the behavioral data were controlled with the "Presentation" software (Neurobehavioral Systems, Version 13.0). Before the scanning session and outside the scanner a presentation of the experiment was given, in order to ascertain that the participants understood the instructions. Additionally, after each scanning session questions were asked in order to assess the tactile, spatial and rhythmic difficulties of the imagery task by means of three items (tactile, How did the grip feel while swinging the club? $1=$ could not feel my two hands $-7=$ hands were formed as a unit; spatial, How did the position of the club's face feel? 1 = could not feel the club's 
face- $6=$ could feel the club's face during swing and its position after impact; rhythm, How was the rhythm of the club's face? $1=$ could not imagine rhythm $-7=$ could feel the backswing, the top swing, the acceleration in the down swing, and the follow through).

\section{DATA ACOUISITION}

The MRI data were acquired on a 3T Philips Intera wholebody magnetic resonance tomograph equipped with an eightchannel Philips SENSE head coil. Whole brain anatomical images were obtained from 160 slices by using a T1-weigthed threedimensional gradient echo pulse sequence (turbo field echo TFE). The following acquisition parameters were used: repetition time $\mathrm{TR}=8 \mathrm{~ms}$, echo time $\mathrm{TE}=3.7 \mathrm{~ms}$, flip angle $=8^{\circ}$, field of view $\mathrm{FOV}=240 \times 240$, slices $=160$, spatial resolution $=$ $0.94 \times 0.94 \times 1 \mathrm{~mm}^{3}$. None of subjects had to be excluded from the study due to anatomical anomalies. Functional data were obtained from 34 slices with a T2* -weighted gradient echo-planar imaging (EPI) sequence, which is sensitive to blood oxygenation level-dependent (BOLD) contrasts. The following acquisition parameters were used: $\mathrm{TR}=2100 \mathrm{~ms}, \mathrm{TE}=35 \mathrm{~ms}$, flip angle $=$ $77^{\circ}, \mathrm{FOV}=220 \times 136 \times 220$, acquisition matrix $=80 \times 80$, voxel size $=2.75 \times 2.75 \times 4.0 \mathrm{~mm}$, slice thickness $4.0 \mathrm{~mm}$, sensitivity encoded (SENSE).

\section{BEHAVIORAL DATA ANALYSIS}

The statistical analysis of the imagery duration was performed with repeated-measures ANOVA (within-subject factor "time" and between-subject factor "group"). The items, concerning the imagery capability, were statistically analyzed using the KruskalWallis test.

\section{fMRI DATA ANALYSIS}

Artifact minimization and $\mathrm{fMRI}$ data analysis was performed with the SPM8 software package (http://www.fil.ion.ucl.ac.uk/spm/) running on MATLAB R2010a (Mathworks Inc., MA, USA). The following preprocessing steps were performed: (1) realignment to the first volume in order to correct for movement artifacts, (2) normalization to the standard stereotactic space (EPI template provided by the Montreal Neurological Institute), (3) smoothing with a Gaussian kernel of $8 \mathrm{~mm}$ full-width-at-half-maximum.

Activated voxels were identified by the general linear model approach, implemented in SPM8. At first-level analysis, for each participant a model with two sessions (T1, T2), three condition regressors (video, imagery, button press movement) and six movement regressors (with the realignment parameters) was aligned. The video regressor was modeled as an epoch lasting $9.7 \mathrm{~s}$ with the onsets of the video sequence. The duration of the imagery epoch was defined by the difference of the two key presses, indicating the start- and end-point of the imagery phase. In order to disentangle the purely motor activity of the motor response induced by the key press, an additional regressor was included in the model. This regressor (i.e., response) bases on the onsets of the second key-press (indicating the end of the imagery phase) and was modeled as an event. A high-pass filter with a cut-off of $128 \mathrm{~s}$ was applied to eliminate slow signal drifts. We were particularly interested in the following contrasts: motor imagery versus response (motor imagery contrast; subsequently abbreviated with MI) and video versus baseline (video contrast; subsequently abbreviated with V). To permit population-level inferences random effect analyses were applied. Thereby, maps of contrast coefficients for each of the first level contrasts were collectively submitted to three analyses of variance (ANOVAs). Two One-Way ANOVAs with the between-subject factor group (golf group, control group) were performed-for the video contrast $\mathrm{V}$ at time-point 1 and for the motor imagery contrast MI at time-point 1 . In order to investigate the training-induced changes of functional recruitment during motor imagery MI, a repeatedmeasures ANOVA (i.e., flexible factorial model in SPM8) with within-subject factor time (T1, T2) and between-subject factor group (golf group, control group) was performed. The $t$-maps resulting from the two One-Way ANOVAs were analyzed at a cluster threshold of $p<0.001$ and FWE (Family-Wise-Error)corrected at cluster level. Clusters from the repeated-measures ANOVA were reported if they survived the significance threshold of $p<0.001$ (uncorrected) with a spatial extent of $k=30$ voxels. The cluster locations were labeled using the Julich Histological atlas.

In order to ascertain that changes in the hemodynamic response seen in the golf group can exclusively be ascribed to the golf training, we performed an additional interaction analysis. This analysis bases on regions-of-interest (ROIs) extracted from the clusters showing a significant change between the two measurement time-points in the golf group. Accordingly, we created ROIs by using the MarsBaR tool (http://marsbar.sourceforge. net/) and the center coordinates were defined by the local maxima of the repeated measures ANOVA (i.e., significant changes between the two measurement time-points in the golf group). We calculated for each ROI and for each subject the mean betaestimates during the motor imagery condition (i.e., MI contrast images). The extracted values were further analyzed using SPSS19 (http://www.spss.com). A repeated-measures ANOVA was calculated with the within-subject factor "time" and the betweensubject factor "group."

\section{RESULTS \\ BEHAVIORAL DATA}

Variations in the overall imagery duration were investigated by means of repeated-measures ANOVA with the within-subject factor "time" and the between-subject factor "group." This analysis neither showed a significant interaction effect nor a significant main effect. Furthermore, after each scanning session participants rated the difficulty to imagine a golf swing by means of three items. At both time-points and for each item, the ratings did not differ significantly between the two groups (Table $\mathbf{1}$ ).

\section{fMRI RESULTS: NEURONAL ACTIVITY IN THE EXPERIMENTAL CONDITIONS (MI, V) AT T1}

In a first step, we were interested in the hemodynamic response during the two conditions (MI, V) at T1 for both groups. This analysis bases on the whole brain data. During the imagery condition MI activity was mainly observed in secondary motor areas, sub-cortical regions (nucleus caudatus), as well as the superior parietal cortex (Figure 1 and Table 2). Independent of group, 
Table 1 | Self-report of the imagery competence.

\begin{tabular}{llll}
\hline & Group & $\boldsymbol{M}$ & SD \\
\hline Tactile T1 & Golf & 4.0 & 2.4 \\
& Control & 4.2 & 2.3 \\
Tactile T2 & Golf & 6.0 & 1.2 \\
& Control & 5.5 & 1.1 \\
Spatial T1 & Golf & 2.9 & 1.4 \\
& Control & 3.2 & 1.9 \\
Spatial T2 & Golf & 4.5 & 1.7 \\
& Control & 3.8 & 1.5 \\
Rhythm T1 & Golf & 4.7 & 2.3 \\
& Control & 5.6 & 1.7 \\
Rhythm T2 & Golf & 5.6 & 1.9 \\
& Control & 5.1 & 2.1 \\
& & &
\end{tabular}

Imagery competence scores for each group at each time-point. the video condition $\mathrm{V}$ was associated with an increased hemodynamic response in vision relevant regions, in particular the bilateral occipital cortex. Along with the occipital cortex, this cluster included also the superior parietal lobule. In addition, the ventral and dorsal PMC (bilateral) showed to be involved while participants watched a slow-motion golf swing movement (Table 3).

\section{fMRI RESULTS: TRAINING-INDUCED CHANGES IN FUNCTIONAL RECRUITMENT DURING MI}

Significant changes of neuronal recruitment were only observed in the golf group and not in the control group. While mentally rehearsing a golf swing, the golf group demonstrated a significant decrease of neuronal recruitment from the first to the second measurement time-point (i.e., T1 > T2), in the right and left dorsal premotor cortex (Table 4 and Figure 2). The other tests investigating changes between the two time-points, i.e., increases

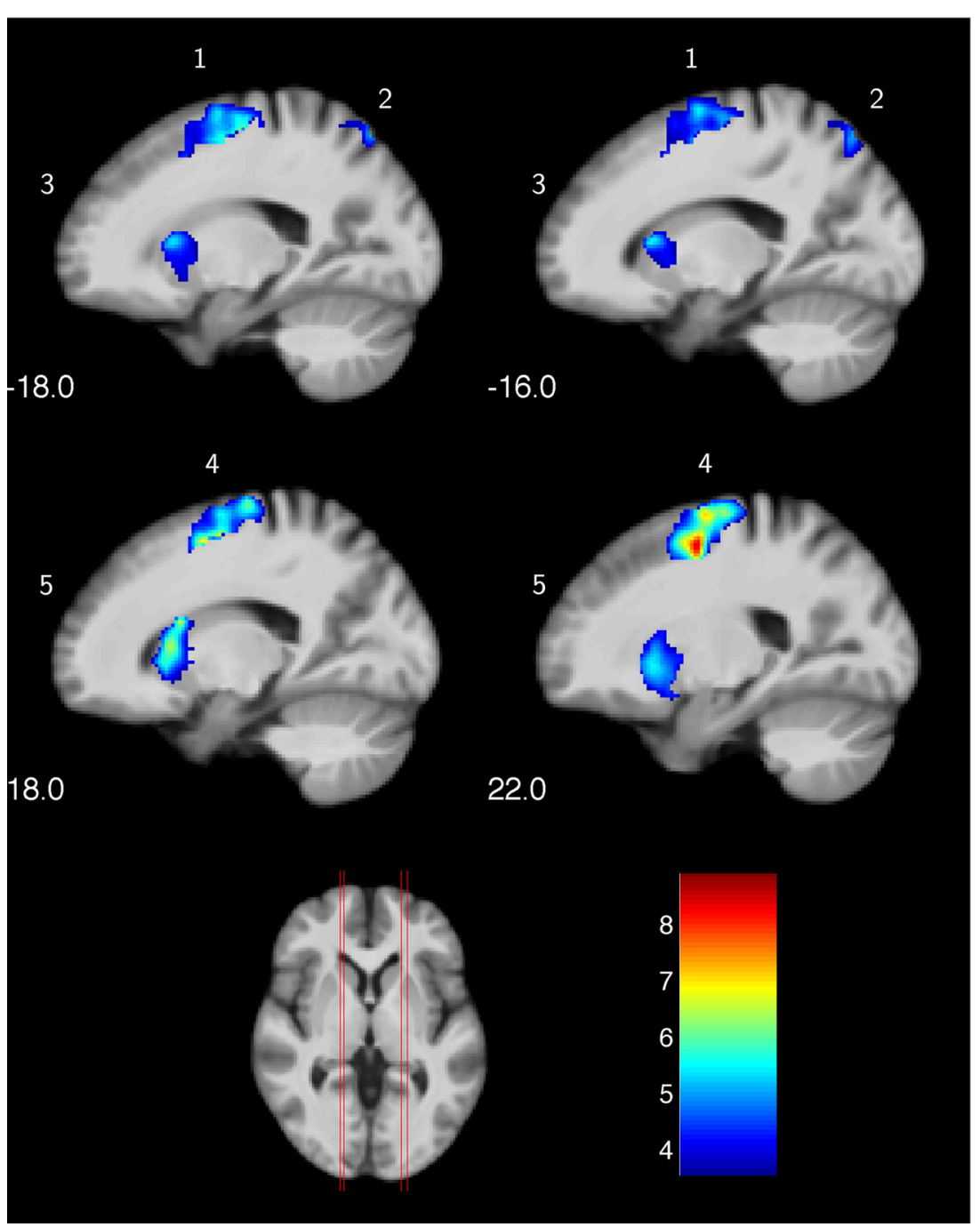

FIGURE 1 | Increased hemodynamic response while mentally rehearsing a golf swing (MI) in the golf and control group at baseline (T1). (1) left premotor cortex; (2) left superior parietal lobe; (3) left nucleus caudatus; (4) right premotor cortex (5) right nucleus caudatus. The color bar represents the $T$-values. 
Table 2 | Motor imagery contrast (MI) at T1.

\begin{tabular}{|c|c|c|c|c|c|c|c|}
\hline Anatomical region & Hem. & \multicolumn{3}{|c|}{ MNI } & $T$ & $\boldsymbol{k}_{E}$ & $p<$ \\
\hline Nucleus caudatus & $\mathrm{R}$ & 18 & 12 & 18 & 6.5 & 702 & 0.001 \\
\hline Nucleus caudatus & $L$ & -16 & 16 & 8 & 5.64 & 527 & 0.001 \\
\hline SPL/Precuneus & $\mathrm{L}$ & -18 & -74 & 56 & 5.25 & 479 & 0.001 \\
\hline
\end{tabular}

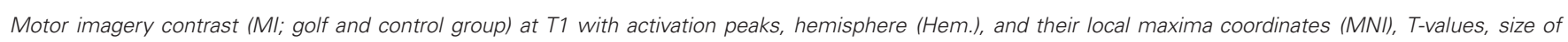
respective cluster $\left(k_{E}\right)$, and significance level. $A P M C$, dorsal premotor cortex; SMA, supplementary motor area; SPL, superior parietal lobe.

Table 3 | Video contrast (V) at T1.

\begin{tabular}{|c|c|c|c|c|c|c|c|}
\hline Anatomical region & Hem. & \multicolumn{3}{|c|}{ MNI } & $T$ & $k_{E}$ & $p<$ \\
\hline Middle occipital gyrus & $\mathrm{L}$ & -20 & -96 & -4 & 16.52 & 28106 & 0.001 \\
\hline VPMC & $\mathrm{R}$ & 44 & 0 & 52 & 6.73 & 2016 & 0.001 \\
\hline dPMC & $\mathrm{L}$ & -28 & -4 & 52 & 7.56 & 1272 & 0.001 \\
\hline Hippocampus & $\mathrm{R}$ & 20 & -32 & 0 & 8.72 & 312 & 0.001 \\
\hline
\end{tabular}

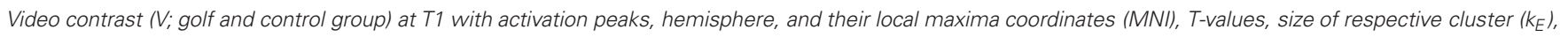
and significance level. $A P M C$, dorsal premotor cortex; VPMC, ventral premotor cortex.

Table 4 | Training-induced decrease (T1 > T2) of functional recruitment during MI.

\begin{tabular}{|c|c|c|c|c|c|c|c|c|}
\hline Number & Anatomical region & Hem. & \multicolumn{3}{|c|}{ MNI } & $T$ & $\boldsymbol{k}_{E}$ & $p<$ \\
\hline 2 & dPMC & $\mathrm{R}$ & 28 & -14 & 58 & 5.31 & 58 & 0.001 \\
\hline 3 & dPMC & $\mathrm{R}$ & 16 & -18 & 70 & 4.69 & 34 & 0.001 \\
\hline 4 & $\mathrm{dPMC}$ & $\mathrm{L}$ & -20 & -12 & 54 & 4.49 & 33 & 0.001 \\
\hline
\end{tabular}

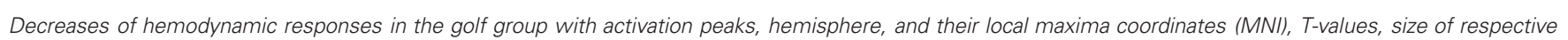
cluster ( $\left.k_{E}\right)$, and significance level. $d P M C$, dorsal premotor cortex. The indices relate to the numbers used in Figures $\mathbf{2}$ and $\mathbf{3}$.

during MI as well as decreases and increases during the video condition, did not differ with respect to neuronal recruitment patterns. Equally as the previous section, these results base on the analysis of whole brain data.

\section{INTERACTION ANALYSIS "GROUP X TIME-POINT"}

The changed hemodynamic response in the golf group during MI was further confirmed by the interaction analysis (Figure 3) with the ROI-approach. In the right and left PMC the golf group demonstrated significant decreases of activation, while significant changes in the control group were absent.

\section{DISCUSSION}

The present longitudinal study sought to delineate the effects of golf training on neural recruitment patterns associated with mentally rehearsing a golf swing. As hypothesized, we observed reduced hemodynamic responses during motor imagery of the golf swing at post-compared with pre-training measurements. These changes in neural recruitment were seen in task-relevant cortical regions and were limited to the golf group. In the following, we first discuss the general pattern of activation seen in both groups at pre-training measurements and then address the issue of training-induced changes.

NEURAL UNDERPINNINGS OF MENTALLY REHEARSING A GOLF SWING

While mentally rehearsing a golf swing, subjects of both groups (having no or very little previous golfing experience) demonstrated neural activity in a large network including cortical and sub-cortical sensorimotor regions and in areas of the dorsal stream. This finding agrees nicely with previous research on the neuronal underpinnings of motor imagery (Jancke et al., 2001; Lotze and Halsband, 2006). These demonstrated that secondary cortical motor areas (e.g., premotor cortex and supplementary motor area) and the parietal lobe were strongly involved in the preparation of mental movements that were not executed overtly. Especially novices or subjects with a low skill level have been shown to recruit a large neural circuit while mentally rehearsing the untrained movement (Ross et al., 2003; Milton et al., 2007; 


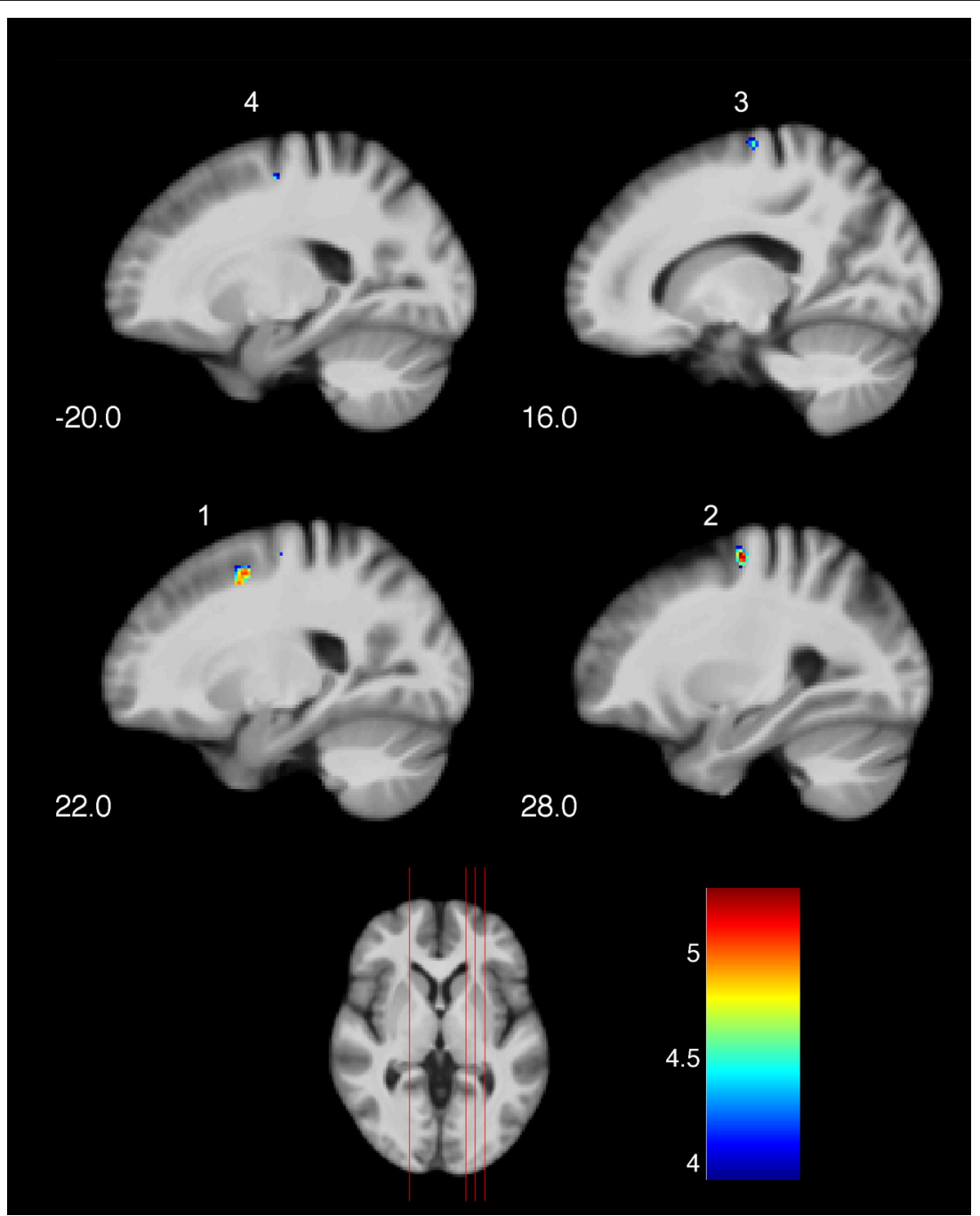

FIGURE 2 | Training-induced changes of neuronal recruitment while mentally rehearsing a golf swing (MI). (1), (2), (3) right premotor cortex; (4) left premotor cortex. The color bar represents the $T$-values. The indices relate to the numbers used in Table $\mathbf{4}$ and Figure $\mathbf{3}$.

Olsson et al., 2008a). Consistent with the activation of the basal ganglia, several of these subcortical nuclei have been repeatedly shown to play a critical role during the process of skill learning, especially when a new motor sequence has to be learned (Doyon et al., 2009).

\section{TRAINING-INDUCED CHANGES}

The results of the repeated-measure ANOVA for comparing preand post-training activation patterns indicate changes of taskrelated brain activation in the golf group, while this effect was not observed in the control group. Specifically, we found a reduction of task-related brain activation from pre- to post-training predominately in the right dorsal premotor cortex.

Our finding of reduced activation at post-training measurement is well in line with previous studies on the neuronal underpinnings of motor expertise and motor skill learning. Milton et al. (2007), for example, demonstrated that the organization of the involved neuronal network during the pre-shot routine of a golf swing differs depending on the level of expertise. Compared with golf novices, golf professionals recruited a smaller and more focused neuronal network during the preparatory phase that precedes the golf swing. Further evidence that the training effect is reflected in a decrease of brain activation while performing expertise-similar movements is provided by cross-sectional studies with highly trained experts in other domains, such as musicianship (Jancke et al., 2000; Koeneke et al., 2004). But previous studies also suggest that the phenomenon of training-induced reductions in neural activity is not exclusively tied to the extreme amount of practice seen in professional athletes or musicians. There is little evidence from longitudinal neuroimaging studies in novices that a reduction of the hemodynamic response might occur already at early stages of learning, that is, after a few weeks of real or mental practice (Hlustik et al., 2004; Koeneke et al., 2006; Ma et al., 2010). However, the pertinent literature does not allow clear conclusions regarding the time-point of transition from the initial phase of activation increase (Karni et al., 1995; 


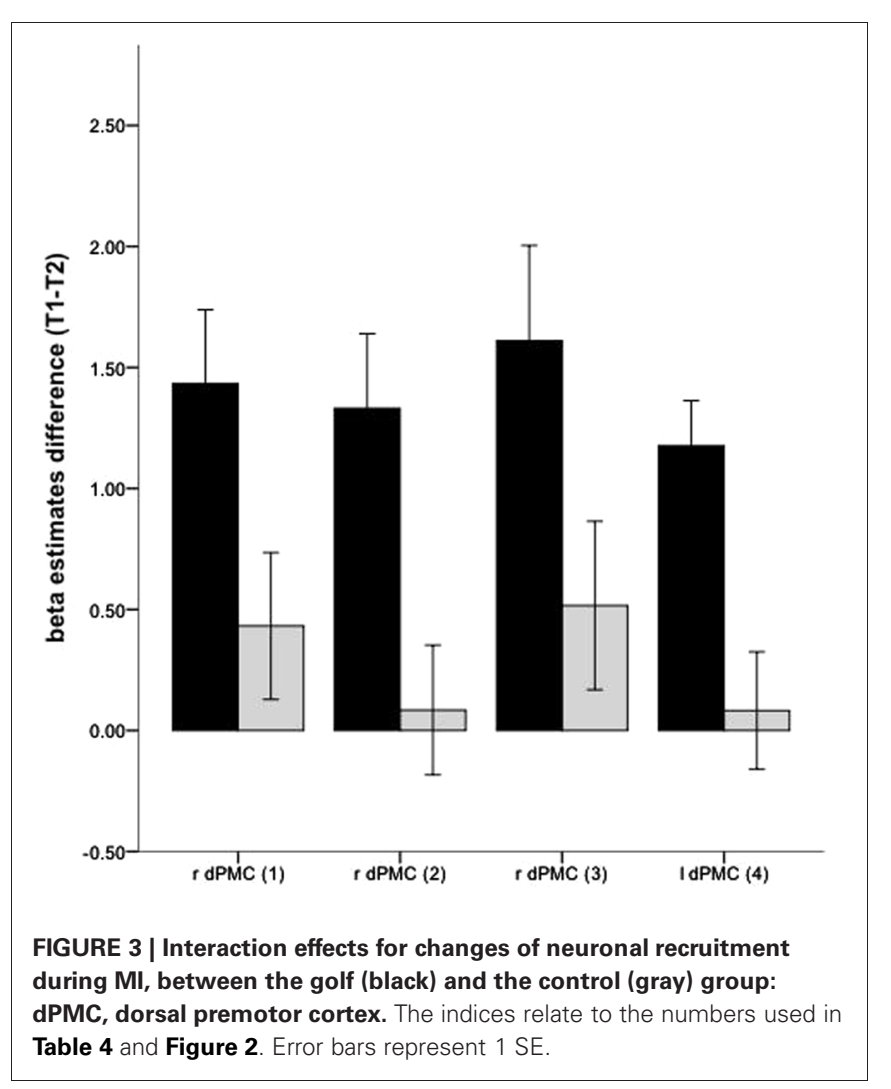

Hlustik et al., 2004) to the phase where activation drops below the pre-training level.

We suggest that the observed decrease of neuronal activation is explained by a training-induced increase in neuronal efficacy. Based on the preceding findings, it was hypothesized that individuals execute or prepare a well-trained (as a consequence of practice) motor task more efficiently, with less effort, and with less degrees of freedom (Bernstein, 1967; Jancke et al., 2000). The latter point refers to the theoretical view that a higher skill level is accompanied by more integrative motor control. Through experience this control process makes use of synergies by linking different effectors (e.g., number of limbs, joints, etc.) together functionally-meaning that more effectors may be supervised simultaneously without the need for separate control of each effector (Tuller et al., 1982). Concerning the decrease of neuronal activity in the premotor cortex, we assume that golf training influences motor control processes of the golf swing by reducing the number of degrees of freedom. Accordingly, the golf group might require less neural effort to mentally control the golf swing at post-training measurement. We further hypothesize that skill improvement and the associated increase in motor control efficiency impacts on both types of movement - the execution of the real golf swing and the mental rehearsal of the golf swing.

Increased competence in imagery at post-measurement may provide an alternative explanation for reduced hemodynamic response at post-measurement. This assumption is based on work showing that participants with better imagery performance do so in association with a less extended recruitment pattern (Guillot et al., 2008). The fact that the golf novices did not rate the imagery competence differently between the two measurement time-points argues for a minor role of this factor in the present study. But our self-report measure of imagery competence is of course only a rough indicator that might not entirely reflect true behavior (this being a common drawback of imagery studies). Given that these two explanations are not mutually exclusive, a combination of both explanations might be relevant for the present findings.

In the present study, a decrease of neuronal recruitment was primarily located in the right hemisphere. Learning-related dynamic processes in the two hemispheres might be considered a possible explanation of this lateralization effect. Motor learning is known to induce a decrease of activation in areas belonging to the right hemisphere, whereas areas belonging to the left hemisphere rather show an increase of neuronal involvement (Serrien et al., 2006). In accordance with this dynamic view of hemispheric particularities, the study by Debaere et al. (2004) demonstrated learning-related activation decreases in right-hemispheric regions, including the right premotor cortex, over a four-day course of complex hand training (Debaere et al., 2004). In contrast, areas belonging to the left hemisphere showed a more prominent role at later stages of the learning process. The authors suggested that changes in task complexity or attention might induce a take-over from the right to left hemisphere. But given the highly complex imagery task and the unusual environment (i.e., scanner noise and supine body position), we doubt that the reduced involvement of the right premotor cortex can be explained by less attentional demands in the present study. Instead, changes in task complexity may be related to the suggestion of a more efficient network and thus explain the present functional plastic adaptations in the golf group. Clearly, future research is needed to disentangle these factors (e.g., attentional demands, task complexity, and imagery competence) that potentially influence this training-induced improved neuronal network.

In contrast to the previous studies (mentioned above) based on very simple motor tasks such as finger tapping or finger sequence learning, the present golf motor training consisted of complex movements. Learning to play golf puts high demands on motor and cognitive control processes that are necessary to prepare the golf swing, accomplish the eye-body coordination, and control movements of different body parts with exact timing. Importantly, training was performed as a true leisure activity with highly individual training protocols and parameters. In contrast, previous studies have attached great importance to keeping training parameters constant across subjects in order to optimize effect strengths (Lustig et al., 2009). To the best of our knowledge, our mental rehearsal study is the first to demonstrate the induction of robust effects at the level of neural activity by a true leisure activity. Thus, the present study expands our knowledge about training-induced neuroplasticity by demonstrating effects of a motor training that has an improved ecological validity.

A further new aspect of the present study relates to the age of the studied cohort. To date, use of motor imagery to examine the neural mechanisms of motor learning has focused primarily on young study populations. However, behavioral studies show substantial age differences during the process of motor learning, 
specifically in terms of age-distinct learning curves (Verwey, 2010; Verwey et al., 2011). So far, the question of age differences in the neuronal representation of motor imagery has not been addressed. Although the present study with its relatively small group size has not the appropriate design to answer this question, the present findings do indicate that participants in their middle adulthood show comparable neuronal recruitment patterns as younger participants in previous studies (Szameitat et al., 2007). Furthermore, the present results demonstrate that training-induced functional plasticity is not a process limited to young adults, thus providing further evidence that brain adaptations due to skill learning can occur across the whole life span-also in middle adulthood. Future intervention studies with different age groups (young adults, middle-aged adults, and old adults) or studies including a larger sample size are needed in order to shed more light on this issue.

\section{CONCLUSION}

Taken together, our results extend previous research in various aspects. Our findings indicate that $40 \mathrm{~h}$ of training of a complex physical leisure activity induces functional neuroplasticity. This process is reflected in a pre-post training reduction

\section{REFERENCES}

Annett, M. (1970). The contribution of the insula to motor aspects of speech production: a review and a hypothesis. Brain Lang. 89, 320-328.

Bernstein, N. (1967). The Coordination and Regulation of Movements. London: Pergamon Press.

Bezzola, L., Merillat, S., Gaser, C., and Jancke, L. (2011). Training-induced neural plasticity in golf novices. J. Neurosci. 31, 12444-12448.

Debaere, F., Wenderoth, N., Sunaert, S., Van Hecke, P., and Swinnen, S. P. (2004). Changes in brain activation during the acquisition of a new bimanual coodination task. Neuropsychologia 42, 855-867.

Doyon, J., Bellec, P., Amsel, R., Penhune, V., Monchi, O., Carrier, J., Lehericy, S., and Benali, H. (2009). Contributions of the basal ganglia and functionally related brain structures to motor learning. Behav. Brain Res. 199, 61-75.

Doyon, J., and Benali, H. (2005). Reorganization and plasticity in the adult brain during learning of motor skills. Curr. Opin. Neurobiol. $15,161-167$.

Draganski, B., and May, A. (2008). Training-induced structural changes in the adult human brain. Behav. Brain Res. 192, 137-142.

Fourkas, A. D., Bonavolonta, V., Avenanti, A., and Aglioti, S. M. (2008). Kinesthetic imagery and tool-specific modulation of corticospinal representations in expert tennis players. Cereb. Cortex 18, 2382-2390.
Gerardin, E., Sirigu, A., Lehericy, S., Poline, J. B., Gaymard, B., Marsault, C., Agid, Y., and Le Bihan, D. (2000). Partially overlapping neural networks for real and imagined 1093-1104.

Godde, B., and Voelcker-Rehage, C. (2010). More automation and less cognitive control of imagined walking movements in high- versus low-fit older adults. Front. Aging Neurosci. 2:139. doi: 10.3389/fnagi.2010.00139

Granert, O., Peller, M., Gaser, C., Groppa, S., Hallett, M., Knutzen, A., Deuschl, G., Zeuner, K. E., and Siebner, H. R. (2011). Manual activity shapes structure and function in contralateral human motor hand area. Neuroimage 54, 32-41.

Guillot, A., Collet, C., Nguyen, V. A., Malouin, F., Richards, C., and Doyon, J. (2008). Functional neuroanatomical networks associated with expertise in motor imagery. Neuroimage 41, 1471-1483.

Halsband, U., and Lange, R. K. (2006). Motor learning in man: a review of functional and clinical studies. J. Physiol. Paris 99, 414-424.

Hlustik, P., Solodkin, A., Noll, D. C., and Small, S. L. (2004). Cortical plasticity during three-week motor skill learning. J. Clin. Neurophysiol. 21, 180-191.

Ilg, R., Wohlschlager, A. M., Gaser, C., Liebau, Y., Dauner, R., Woller, A., Zimmer, C., Zihl, J., and Muhlau, M. (2008). Gray matter increase induced by practice correlates with hand movements. Cereb. Cortex 10,

in neuronal activity in task-relevant cortical areas during the mental rehearsal of a golf swing. Furthermore, this applies for the population of middle-aged adults that has as yet received scare attention in research. These findings support the idea that improved skill is associated with greater efficiency in associated neuronal networks. The training-induced improvement in skill appears to influence the neural processes associated with imagery of practiced movements. Finally, our findings suggest that motor training protocols characterized by a low-to-moderate training intensity and a highly individual training schedule, and therefore by high ecological validity, are effective in inducing functional neuroplastic changes.

\section{ACKNOWLEDGMENTS}

This project was supported by the Research Funding of the University of Zurich (56234103), a project grant from the "Bundesamt für Sport" (36234110), and the International Normal Aging and Plasticity Imaging Center (INAPIC) University of Zurich. Ladina Bezzola is a fellow of the International Max Planck Research School on the Life Course (LIFE). The authors thank Marcus Cheetham for providing English language proofreading.

task-specific activation: a combined functional and morphometric magnetic resonance imaging study. J. Neurosci. 28, 4210-4215.

Ionta, S., Ferretti, A., Merla, A., Tartaro, A., and Romani, G. L. (2010). Stepby-step: the effects of physical practice on the neural correlates of locomotion imagery revealed by fMRI Hum. Brain Mapp. 31, 694-702.

Jancke, L. (2009). The plastic human brain. Restor. Neurol. Neurosci. 27, 521-538.

Jancke, L., Kleinschmidt, A., Mirzazade, S., Shah, N. J., and Freund, H. J. (2001). The role of the inferior parietal cortex in linking the tactile perception and manual construction of object shapes. Cereb. Cortex 11, 114-121.

Jancke, L., Shah, N. J., and Peters, M. (2000). Cortical activations in primary and secondary motor areas for complex bimanual movements in professional pianists. Brain Res. Cogn. Brain Res. 10, 177-183.

Jeannerod, M. (2001). Neural simulation of action: a unifying mechanism for motor cognition. Neuroimage 14, S103-S109.

Karni, A., Meyer, G., Jezzard, P., Adams, M. M., Turner, R., and Ungerleider, L. G. (1995). Functional MRI evidence for adult motor cortex plasticity during motor skill learning. Nature 377, 155-158.

Koeneke, S., Lutz, K., Esslen, M., and Jancke, L. (2006). How finger tapping practice enhances efficiency of motor control. Neuroreport 17, 1565-1569.
Koeneke, S., Lutz, K., Wustenberg, T., and Jancke, L. (2004). Longterm training affects cerebellar processing in skilled keyboard players. Neuroreport 15, 1279-1282.

Lacourse, M. G., Orr, E. L., Cramer, S. C., and Cohen, M. J. (2005). Brain activation during execution and motor imagery of novel and skilled sequential hand movements. Neuroimage 27, 505-519.

Lotze, M., and Halsband, U. (2006). Motor imagery. J. Physiol. Paris 99, 386-395.

Lustig, C., Shah, P., Seidler, R., and Reuter-Lorenz, P. A. (2009). Aging, training, and the brain: a review and future directions. Neuropsychol. Rev. 19, 504-522.

Ma, L., Wang, B., Narayana, S., Hazeltine, E., Chen, X., Robin, D. A., Fox, P. T., and Xiong, J. (2010). Changes in regional activity are accompanied with changes in inter-regional connectivity during 4 weeks motor learning. Brain Res. 1318, 64-76.

Milton, J., Small, S. L., and Solodkin, A. (2008). Imaging motor imagery: methodological issues related to expertise. Methods 45, 336-341.

Milton, J., Solodkin, A., Hlustik, P. and Small, S. L. (2007). The mind of expert motor performance is cool and focused. Neuroimage 35, 804-813.

Olsson, C. J., Jonsson, B., Larsson, A., and Nyberg, L. (2008a). Motor representations and practice affect brain systems underlying imagery: an fMRI study of internal 
imagery in novices and active high jumpers. Open Neuroimag. J. 2, 5-13.

Olsson, C. J., Jonsson, B., and Nyberg, L. (2008b). Learning by doing and learning by thinking: an fMRI study of combining motor and mental training. Front. Hum. Neurosci. 2:5. doi: 10.3389/neuro.09.005.2008

Page, S. J., Szaflarski, J. P., Eliassen, J. C., Pan, H., and Cramer, S. C. (2009). Cortical plasticity following motor skill learning during mental practice in stroke. Neurorehabil. Neural Repair 23, 382-388.

Pascual-Leone, A., Amedi, A., Fregni, F., and Merabet, L. B. (2005). The plastic human brain cortex. Annu. Rev. Neurosci. 28, 377-401.

Ross, J. S., Tkach, J., Ruggieri, P. M., Lieber, M., and Lapresto, E. (2003). The mind's eye: functional MR imaging evaluation of golf motor imagery. AJNR Am. J. Neuroradiol. 24, 1036-1044.

Rovio, S., Kareholt, I., Helkala, E. L., Viitanen, M., Winblad, B., Tuomilehto, J., Soininen, H., Nissinen, A., and Kivipelto, M.
(2005). Leisure-time physical activity at midlife and the risk of dementia and Alzheimer's disease. Lancet Neurol. 4, 705-711.

Seidler, R. D. (2007). Older adults can learn to learn new motor skills. Behav. Brain Res. 183, 118-122.

Serrien, D. J., Ivry, R. B., and Swinnen, S. P. (2006). Dynamics of hemispheric specialization and integration in the context of motor control. Nat. Rev. Neurosci. 7, 160-166.

Solodkin, A., Hlustik, P., Chen, E. E., and Small, S. L. (2004). Fine modulation in network activation during motor execution and motor imagery. Cereb. Cortex 14, 1246-1255.

Studer, B., Koeneke, S., Blum, J., and Jancke, L. (2010). The effects of practice distribution upon the regional oscillatory activity in visuomotor learning. Behav. Brain Funct. 6, 8.

Szameitat, A. J., Shen, S., and Sterr, A. (2007). Motor imagery of complex everyday movements. An fMRI study. Neuroimage 34, 702-713.
Tuller, B., Turvey, M. T., and Fitch, H. L. (1982). "The Bernstein perspective. II. The concept of muscle linkage or coordinative structure," in Human Motor Behavior, ed J. A. S. Kelso. (Hillsdale, NJ, London: Lawrence Erlbaum Associates), 239-252.

Ungerleider, L. G., Doyon, J., and Karni, A. (2002). Imaging brain plasticity during motor skill learning. Neurobiol. Learn. Mem. 78, 553-564.

Verghese, J., Lipton, R. B., Katz, M. J., Hall, C. B., Derby, C. A., Kuslansky, G., Ambrose, A. F., Sliwinski, M. and Buschke, H. (2003). Leisure activities and the risk of dementia in the elderly. N. Engl. J. Med. 348, 2508-2516.

Verwey, W. B. (2010). Diminished motor skill development in elderly: indications for limited motor chunk use. Acta Psychol. (Amst.) 134 206-214.

Verwey, W. B., Abrahamse, E. L., Ruitenberg, M. F., Jimenez, L., and De Kleine, E. (2011). Motor skil learning in the middle-aged: limited development of motor chunks and explicit sequence knowledge. Psychol. Res. 75, 406-422.

Conflict of Interest Statement: The authors declare that the research was conducted in the absence of any commercial or financial relationships that could be construed as a potential conflict of interest.

Received: 21 December 2011; paper pending published: 27 January 2012; accepted: 13 March 2012; published online: 29 March 2012.

Citation: Bezzola L, Mérillat $S$ and Jäncke L (2012) The effect of leisure activity golf practice on motor imagery: an fMRI study in middle adulthood. Front. Hum. Neurosci. 6:67. doi: 10.3389/fnhum.2012.00067

Copyright (C) 2012 Bezzola, Mérillat and Jäncke. This is an open-access article distributed under the terms of the Creative Commons Attribution Non Commercial License, which permits noncommercial use, distribution, and reproduction in other forums, provided the original authors and source are credited. 